\title{
Macroscopic response and directional disorder dynamics in chemically substituted ferroelectrics
}

\author{
Jacopo Parravicini, ${ }^{1,{ }^{*}}$ Eugenio DelRe, ${ }^{1}$ Aharon J. Agranat, ${ }^{2}$ and Gianbattista Parravicini ${ }^{3,4}$ \\ ${ }^{1}$ Dipartimento di Fisica, "Sapienza" Università di Roma, 00185 Roma, Italy \\ ${ }^{2}$ Applied Physics Department, Hebrew University of Jerusalem, 91904, Israel \\ ${ }^{3}$ Dipartmento di Fisica, Università di Pavia, 27100 Pavia, Italy \\ ${ }^{4}$ IPCF-CNR, "Sapienza" Università di Roma, 00185 Roma, Italy \\ (Received 1 August 2015; revised manuscript received 7 December 2015; published 21 March 2016)
}

\begin{abstract}
Using temperature-resolved dielectric spectroscopy in the range 25-320 K we investigate the macroscopic response, phase symmetry, and order/disorder states in bulk ferroelectric $\mathrm{K}_{1-y} \mathrm{Li}_{y} \mathrm{Ta}_{1-x} \mathrm{Nb}_{x}$ (KLTN). Four longrange symmetry phases are identified with their relative transitions. Directional analysis of the order/disorder states using Fröhlich entropy indicates global symmetry breaking along the growth axis and an anisotropic dipolar effective thermodynamic behavior, which ranges from disordered to ordered at the same temperature for different directions in the sample. Results indicate that the macroscopic polarization, driven by nanosized polar regions, follows a microscopic perovskite eight-sites lattice model.
\end{abstract}

DOI: 10.1103/PhysRevB.93.094203

\section{INTRODUCTION}

Many promising functional materials with remarkably strong and malleable magnetic, electric, and mechanical properties, are nominally pure crystals that actually host compositional disorder at the nanometric scale [1-4]. Although the presence of disorder does appear to be a key ingredient, its role in response is still largely unknown and subject of intense research efforts [5-7]. One line of research focuses on nanodisordered ferroelectrics, with the aim of developing innovative materials for capacitors, memories, and photonics [8-10]. Among these, an important role is played by solidsolution perovskites, which are commonly accepted as a paradigm of disordered ferroelectric crystals $[5,11,12]$. The behavior of these compounds can continuously span from that of a canonical relaxor, diffuse-transition relaxor, sharptransition relaxor, to a true ferroelectric [5,12], depending on the composition and stoichiometry [5,13]. A key feature of disordered ferroelectrics is that they can manifest many of the properties of standard crystals, in terms of electrical, optical, and mechanical response, and still host a population of nanosized reconfigurable polar regions, the so-called polar nanoregions (PNRs). These are correlated mesoscopic regions of polarizations that are widely considered responsible for the giant electro-optic and piezoelectric effect $[5,14,15]$. Moreover, these PNRs can behave as liquid, solid, and even glassy systems, in the so-called nonergodic dipolar regimes, and all this in one and the same crystal structure, typically a large scale perovskite [16-18]. How exactly a crystal is able to host a dynamic population of dipoles is still an open question, and its understanding evidently lies at the heart of future applications $[5,11,12]$. In fact, it is well known that in a perovskite structure the ion disposition in the $\mathrm{ABO}_{3}$ lattice may be distorted, causing local inherent or internal dipolar disorder $[19,20]$. When a substitutional disorder is simultaneously present, below the so-called Burns temperature $T_{B}$, its combination

\footnotetext{
${ }^{*}$ Corresponding author: jacopo.parravicini@roma1.infn.it; Currently with Dipartimento di Scienza dei Materiali, Università di Milano-Bicocca, 20125 Milano, Italy.
}

with this internal disorder can give rise to lattice distortions, strains, and local electric fields [21], which then lead to PNRs [3,5,22-24]. Moreover, the growth process along a specific direction (say $c$ ) is known to strongly influence the defects and the distributions of strains and charges even though the crystal is never poled [25,26]. Altogether these phenomena are able to cause a local symmetry breaking in what would be normally considered a high-symmetry paraelectric phase, a breaking that generates a wide landscape of PNR-driven properties whose nature and temperature dependence is still under study. The local ordering and crystalline symmetry of PNRs have been extensively investigated using various techniques, the principal being diffractometric [20,27-35]. Results indicate that the behavior and response of PNR-hosting samples is strongly affected by disorder [5,13,21,36,37]. Several works have been devoted to investigating PNR dynamics in correspondence to the cubic-to-tetragonal phase transition using NMR and EXAFS techniques [29-31]. Moreover, also in the lower temperature orthorhombic and rhombohedral phases, the internal symmetry of the PNRs was recently explored [27,28]. Nonetheless the features of the PNRs in the low-temperature crystalline phases remain, at present, only partially explored for a very restricted number of compounds (e.g., $\mathrm{BaTiO}_{3}$, $\mathrm{SrTiO}_{3}, \mathrm{KNbO}_{3}$, and $\left.\mathrm{PbTiO}_{3}\right)[5,11,12,23]$.

Here we study the dielectric response of a solid solution single crystal KLTN, a disordered ferroelectric with established out-of-equilibrium properties and giant response [25,38-40]. Directional components of entropy variations are inspected using dielectric spectroscopy and Fröhlich entropy [41-43] (Fig. 1). We focus on the correlation between the degrees of order (disorder) and four long-range symmetry phases in the temperature range $25-320 \mathrm{~K}$. The anisotropic features are specifically investigated by considering the structural phases along the three main crystalline directions. Remarkably, the distribution of the macroscopic order/disorder state is found to be in agreement with the microscopic so-called eight-sites model developed by Comès et al. [19,20]. This model was introduced to account for diffused photon scattering in $\mathrm{x}$ ray studies of $\mathrm{BaTiO}_{3}$ and $\mathrm{KNbO}_{3}$ and implies that some perovskites never actually form high-temperature crystalline phases, have order/disorder transitions, and have a built-in 


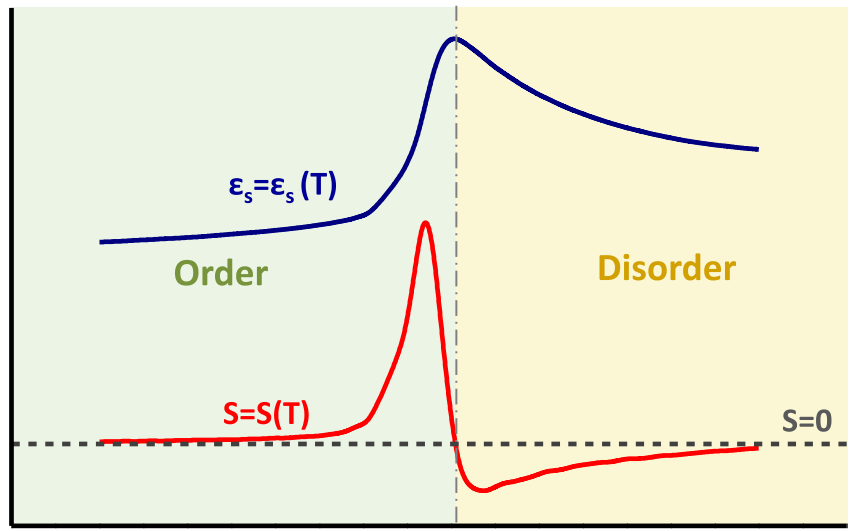

Temperature

FIG. 1. From [41]: Schematic temperature dependence of the dielectric constant $\epsilon_{s}$ (blue curve) and of the related entropy change as defined by Eqs. (1) and (2): $S \propto \partial \epsilon_{S} / \partial T$ (red curve) due to the field-induced polarization. Fröhlich stated that if $S>0$ the field creates disorder, if $S<0$ it creates order.

capability of hosting disorder. Our results bring us to conclude that it is these extra degrees of freedom that support the polar dynamics of the PNRs within a specific crystal phase, a basic tassel in our understanding and harnessing of functional ferroelectrics and relaxors.

\section{EXPERIMENTS}

We have grown a solid solution of lithium-enriched potassium-tantalate-niobate $\left(\mathrm{K}_{1-y} \mathrm{Li}_{y} \mathrm{Ta}_{1-x} \mathrm{Nb}_{x} \mathrm{O}_{3}\right)$ with $x=$ $0.36, y=0.003$, single crystal using the top-seeded-solution method and we have extracted a $c$-direction-pulled zero-cut $3.87^{(a)} \times 3.55^{(b)} \times 2.30^{(c)} \mathrm{mm}$ sample [25,44]. The concentration of potassium, tantalum, and niobium in the KLTN sample was determined by electron microprobe analysis. The concentration of $\mathrm{Cu}$ and $\mathrm{Li}$ were measured using flame atomic absorption spectroscopy employing a procedure that was specially developed for KLTN and described in [45]. The permittivity measurements versus temperature are carried out by a precision LCR meter (Agilent-4284A) for different frequencies, applying a probing $1 \mathrm{~V} / \mathrm{cm}$ field to planeparallel electrodes painted on the crystal facets. Temperature variation in the $25-320 \mathrm{~K}$ range is achieved using a $T$ controlled closed two-stage helium cryostat (in a vacuum configuration). The cooling rate is $0.5 \mathrm{~K} / \mathrm{min}$ and temperature is monitored through a calibrated silicon diode sensor ( 0.01 $\mathrm{K}$ in precision). The efficient thermal contact of the sample with the temperature source and the adopted low cooling rate render each single permittivity $\epsilon$ measurement isothermal. The dielectric response is measured along the three crystalline main directions, $a, b, c$, in three different consecutive scan stages, keeping all experimental parameters constant, in particular thermal cooling rate, applied field value, and thermal contact. After the experimental stage in one direction is accomplished, the pair of parallel electrodes is removed and placed on the facets corresponding to the next direction. Each set of measurements was carried out for the frequencies: 1, 10, 100, $1000 \mathrm{kHz}$.

\section{A. Dielectric response}

In Figs. 2(a)-2(c) the relative permittivity as a function of temperature $\epsilon(T)$ is reported for the employed frequencies along the crystalline axes $a, b$, and $c$, respectively, in the $25-320 \mathrm{~K}$ range, on cooling. The $\epsilon(T)$ curves, for all the investigated directions, give evidence of the occurring of three phase transitions. In particular, considering the $a$ direction [Fig. 2(a)], three marked peaks are displayed with their maxima $T_{m}$, distributed in frequency. Below the maxima, the overlapping of the permittivity curves for the different frequencies, in correspondence with a sharp decrease, marks

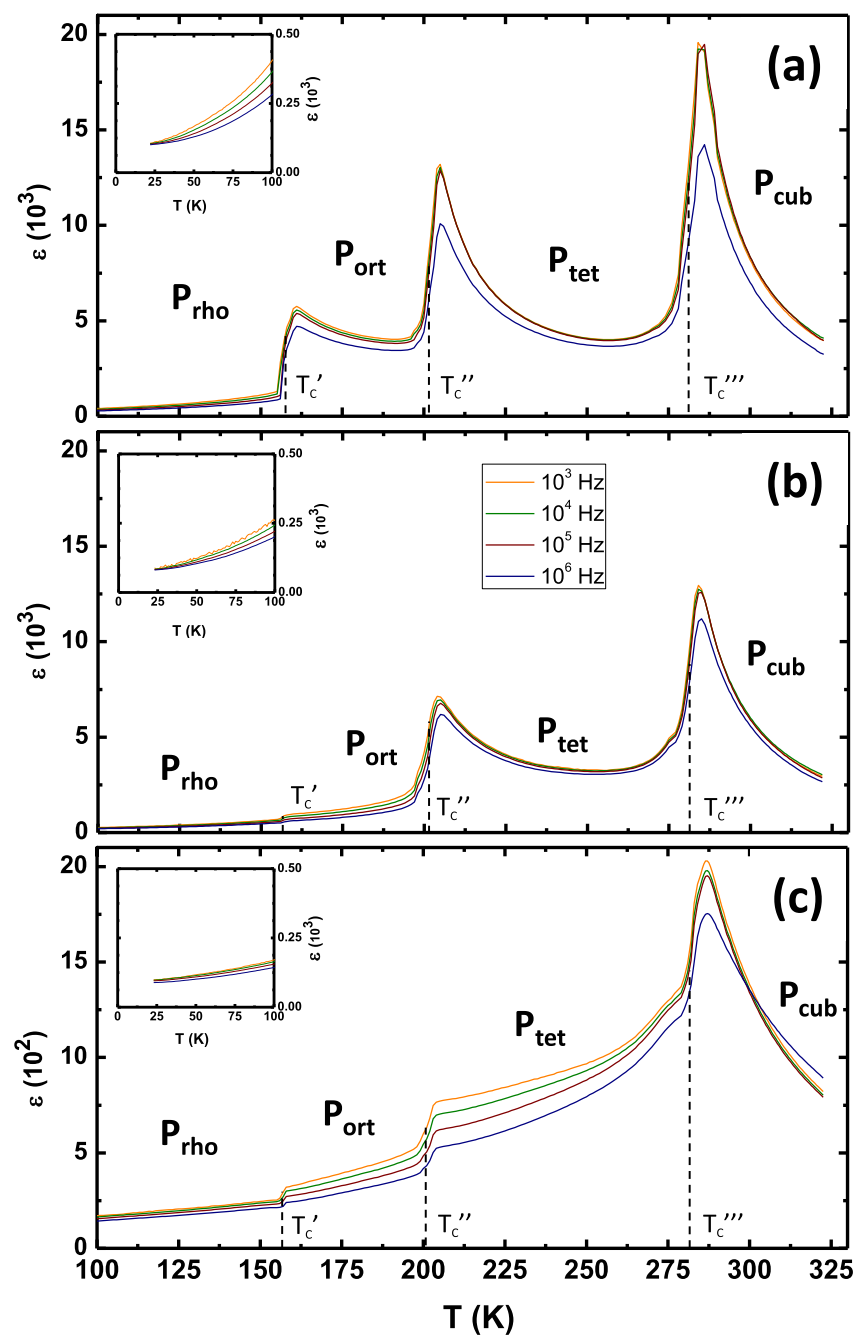

FIG. 2. Dependencies on temperature of the relative dielectric permittivity measured at $10^{3}-10^{6} \mathrm{~Hz}$ frequencies along $a$ (a), $b$ (b), and $c$ (c) directions for the cooling stage. For each plot are indicated the nominal crystalline phases $P_{\text {rho }}$ (rhombohedral), $P_{\text {ort }}$ (orthorhombic), $P_{\text {tet }}$ (tetragonal), and $P_{\text {cub }}$ (cubic), with the relative transition temperatures $T_{C}^{\prime}$ (rhombohedral-to-orthorhombic), $T_{C}^{\prime \prime}$ (orthorhombic-to-tetragonal), and $T_{C}^{\prime \prime}$ (tetragonal-to-cubic). For each transition, the characteristic temperatures are found to be the same for all directions. Note that in main plot (c) the permittivity scale is one order of magnitude smaller than in (a) and (b) plots, while the insets with the same scale show that the differences between permittivities tend to vanish as the temperature approaches $0 \mathrm{~K}$. 
the critical temperature $T_{C}$ of a long-range symmetry transition in the sample $[5,11,12]$. The shift of the permittivity maxima with respect to the corresponding $T_{C}$ and the dispersion in frequency of the $\epsilon(T)$ curves around the maxima signal a broad-type transition. This kind of frequency dependence is found in agreement with that reported in the literature on lead-free relaxor ferroelectrics [5,13]: We note that it does not manifest the features of a canonical relaxor response but instead those of a sharp-transition relaxor [5] (or "incipient relaxor" of [46]).

We associate the detected temperatures $T_{C}^{\prime \prime \prime}=282 \mathrm{~K}, T_{C}^{\prime \prime}=$ $202 \mathrm{~K}$, and $T_{C}^{\prime}=156 \mathrm{~K}$ with the cubic/tetragonal, tetragonal/orthorhombic, and orthorhombic/rhombohedral transitions, respectively, in agreement with other well established disordered perovskite structures [26-28] and with previous studies into different compositions of KLTN [17,25]. We note that dielectric dispersion is detected along the entire range of the four observed structural phases, here indicated as $P_{\text {cub }}, P_{\text {tet }}$, $P_{\text {ort }}, P_{\text {rho }}$ for decreasing temperatures.

Comparing $\epsilon(T)$ in $a, b, c$ directions in the (nominally isotropic) cubic phase $(T>281 \mathrm{~K}$, Fig. 2), we observe different values of the permittivity. Especially along the $c$ axis, i.e., the growth axis, $\epsilon(T)$ is found almost one order of magnitude smaller than in the other directions [Fig. 2(c)]. In the tetragonal phase, which should be symmetric for fields orthogonal to the $c$ axis, i.e., $a$ and $b$ directions should be equivalent, we observe these to have noticeably different permittivity. A similar response is found also for the other lower-temperature crystalline phases and the related directions, except for the lowest (rhombohedral) phase $(T \lesssim$ $50 \mathrm{~K}$ ), where anisotropy tends to vanish (insets of Fig. 2). An analogous behavior (a freezing effect of local domain) can be found, e.g., in [46].

\section{B. Directional analysis using Fröhlich entropy}

The anisotropy in the response is further analyzed in terms of directionally resolved order-disorder states using Fröhlich entropy. This is based on the scheme developed by Fröhlich who formulated the relationship between the behavior of the real part of the dielectric function versus temperature $\epsilon(T)$ and the entropy variation in the system $S=S(T)$. Under suitable conditions the entropy of a dielectric subject to an external electric field $E$ is given, per unit volume, by the relationship $[41,42]$

$$
S(T)=S_{0}(T)+\frac{E^{2} \epsilon_{0}}{2} \frac{\partial \epsilon_{s}}{\partial T}=S_{0}(T)+S_{E}(T),
$$

where $S_{0}$ is the entropy of the system at zero field, $\epsilon_{0}$ is the vacuum permittivity, $\epsilon_{s}$ is the real part of the dielectric function in the low-frequency limit, and $T$ is the absolute temperature. The Fröhlich entropy $S_{E} / E^{2}$ is given by

$$
s(T) \equiv \frac{S_{E}}{E^{2}}=\frac{\epsilon_{0}}{2} \frac{\partial \epsilon_{s}}{\partial T},
$$

which is the field-induced entropy per unit volume and field. Since the electric field selects a specific direction relative to the crystal, $s(T)$ inspects the specific component to the global entropy along the specific direction of the electric field. Equation (2) shows how the application of an electric field increases the entropy if $\partial \epsilon_{s} / \partial T$ is positive, while it decreases entropy when it is negative. As pointed out by Fröhlich, in disordered dipolar systems (which we call "dipolar liquidlike" in analogy with $[17,41])$ an applied electric field creates order since it orients the dipoles otherwise randomly arranged; as a consequence the $s$ entropy variation induced by the field ("Fröhlich entropy") is negative. On the contrary, in dipolar ordered systems (which, similarly to the previous term, we call "dipolar solidlike"), the field perturbs the established ordered state and the fieldinduced entropy $s$ is positive (see Fig. 1). Therefore, the condition $\partial \epsilon_{s} / \partial T>0$ signals a system globally disordered, while when $\partial \epsilon_{s} / \partial T<0$ the system is globally ordered.

When phase transitions are considered, on the other hand, an order/order transition manifests itself as a variation of the positive level of $S_{E}$; on the contrary, an order/disorder transition will manifest itself in the permittivity curve as slope reversal, and in $\partial \epsilon_{s} / \partial T$ as a change from positive to negative sign (Fig. 1) [41-43,47,48]. In Fig. 3 we report the entropy $s=s(T)$ for the $a, b, c$ crystalline directions. In the cubicphase $T$ range [Figs. 3(a)-3(c)], the consistently negative entropy indicates a disorder state for all three measured directions. In turn, in the tetragonal phase a wide interval (206-259 K) of highly disordered state, characterized by the negative value of $s(T)$, is found only in the $a$ and $b$ directions [Figs. 3(a) and 3(b)], while in the $c$ direction [Fig. 3(c)] the induced entropy is positive for the whole range of the phase. Lowering the temperature, in the orthorhombic phase, disorder is found only in the $a$ direction (162-193 K). Finally, in the lowest-temperature rhombohedral phase, the $s(T)$ curve still exhibits a positive sign in the whole $T$ range, for all directions, where it manifests a regular slow decrease with temperature: this response is the signature of a stable ordered phase and characterizes only the rhombohedral symmetry.

\section{DISCUSSION}

\section{A. Permittivity anisotropy}

Permittivity results indicate an anisotropic response with peculiar features. In the highest-temperature region, values of $\epsilon(T)$ as a function of direction evidence anisotropy along the growth axis $c$ also in the nominally centrosymmetric cubic phase (Fig. 2). This suggests a growth-induced alteration in polarization [25], a phenomenon that is commonly associated with internal strain in the pull direction [12]. In specific perovskites this is known to cause the flexoelectric effect, i.e., a macroscopic strain-induced spontaneous polarization which produces the coupling between the strain gradient and polarization [49-51]. Because in a disordered ferroelectric, the strains are inversely proportional to the size of the polar regions $[12,52,53]$, which are nanometric in our case, and the susceptibility has very high values $\left(\epsilon \sim 10^{4}\right.$ and more), we expect this effect be relevant. Congruently, the marked macroscopic breaking of centrosymmetry in the cubic phase (Fig. 2), which was recently demonstrated in perovskite materials caused by flexoelectric dynamics [54], can be recognized as a consistent signature of the presence of this phenomenon. Remarkably, in the lowest region of the rhombohedral phase $(T \lesssim 50 \mathrm{~K})$, when the temperature approaches $0 \mathrm{~K}$, anisotropy tends to vanish (insets of Fig. 2). 


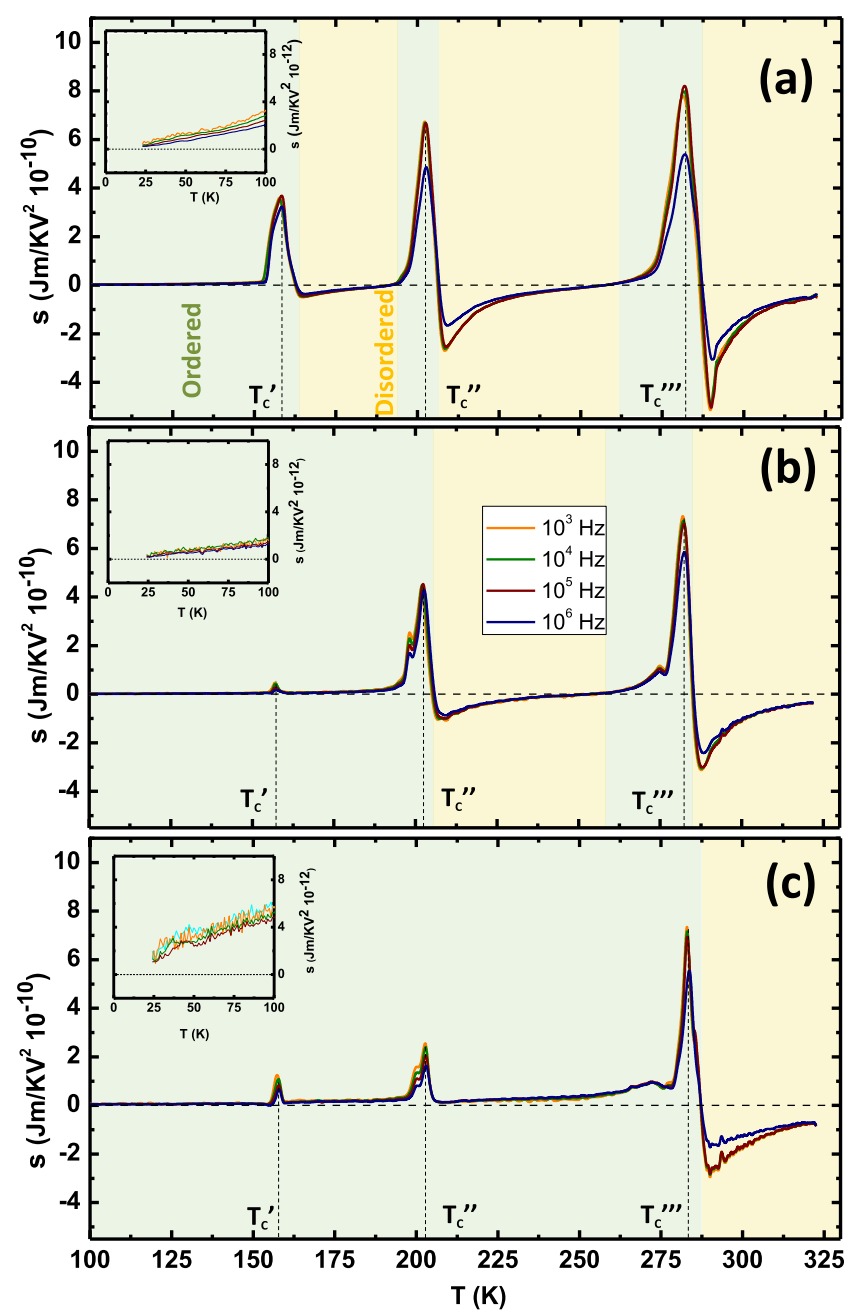

FIG. 3. Frölich entropy [following Eq. (2)] $s_{a}, s_{b}, s_{c}$ for, respectively, $a$ (a), $b$ (b), $c$ (c) axes direction, for unit of volume and field square (cooling stage) in the $100-320 \mathrm{~K}$ temperature range. The indicated temperatures $T_{C}^{\prime}, T_{C}^{\prime \prime}, T_{C}^{\prime \prime}$ are the transition temperatures shown in Fig. 2. The insets show the temperature range 0-100 K for each respective direction and, congruently with the permittivity behavior, they point out that the anisotropy tend to vanish as the temperature approaches $0 \mathrm{~K}$.

\section{B. Order/disorder state anisotropy}

The exploration of the macroscopic order/disorder state of each phase, along the three directions, using Fröhlich entropy differs from the standard diffraction techniques because (i) it is able to give a macroscopic account of the ordering state; (ii) being directly derived from dielectric data, it yields a nearly continuous description of each single phase evolution in temperature, as well as of the transition dynamics $[43,47,48]$; and (iii) it is highly directional, in that it measures entropy in response to an electric field applied in a specific direction relative to the lattice. Here it is worth underlining that, due to the applied probing field strength $(1 \mathrm{~V} / \mathrm{cm})$, entropy data describes the thermal evolution of the system without any coercive (several $\mathrm{kV} / \mathrm{cm}$ ) field effects on the PNR size and local structure, as reported by many authors $[36,55,56]$. The Fröhlich entropy analysis reported in Fig. 3 demonstrates that in the KLTN sample the macroscopic ordering state depends on the long-range symmetry of the phases and, for each phase, on the crystalline directions. Specifically, different (order or disorder) nanosized dipole states can coexist at the same temperature for different directions. Referring to the nominally cubic phase, Fröhlich entropy establishes that the macroscopic PNR-driven dielectric response has features analogous to those of a dipolar liquid (disordered), indicating that PNRs are in a weakly interacting ergodic state, i.e., they act as a liquidlike system being free to reorient in the parent paraelectric matrix $[5,12,13]$.

\section{Eight-sites-model macroscopic response}

Local symmetries and order transitions of the crystal cell have been studied with $\mathrm{x}$-ray diffuse scattering measurements on prototypical perovskite crystals $\mathrm{BaTiO}_{3}$ and $\mathrm{KNbO}_{3}$ by Comès et al. [19,20]. These authors showed that in these compounds an intrinsic disorder is a consequence of the dynamic displacement of the $B$ cation on eight energetically equivalent sites along the diagonals of the cubic cell, whose allowed directions of the displacements are dependent on the given crystalline phase (see the scheme in Fig. 4). Specifically, they assessed that: in the cubic phase there are three planes of diffusive scattering normal to the three cubic axes (i.e., disorder is manifested in all directions); in the tetragonal phase there are only two (disorder is manifested in the directions perpendicular to the tetragonal $c$ axis); in the orthorhombic phase there is only one plane of diffusion. Finally, in the rhombohedral phase there is no diffusion (only this symmetry shows a behavior of a true crystal): in this phase the displacements are correlated and the $B$ cations are displaced in the same direction. Therefore, the rhombohedral phase is found to be the sole ordered and stable phase for the cited perovskites $[19,20,27,28]$. In our specimen, when the order/disorder states are characterized using Fröhlich entropy (Fig. 3), we observe that the distribution of the macroscopic order/disorder states, in relation both with each single phase and crystal directions, is found to be in remarkable agreement with the microscopic so-called eight-sites model developed by Comès and colleagues. From our results we congruently conclude that the macroscopic ordering state throughout all the four phases is essentially driven by this microscopic eight-sites displacements of the $B$ cations through the mesoscopic response of the system of PNRs: these evolve dynamically and occupy the inner states dictated by the Comès model. In particular, our findings show that also in a complex chemical-substituted disordered ferroelectric, similarly to the nominally pure crystals of Comès, a fully ordered state is achieved at the rhombohedral phase. Recent experiments using neutron scattering in

\begin{tabular}{|c|c|c|c|c|}
\hline $\begin{array}{l}\text { Crystalline } \\
\begin{array}{l}\text { Cryytal- } \\
\text { line direction }\end{array} \\
\text { linase }\end{array}$ & $\begin{array}{c}\text { Rhombo } \\
\text { hedral }\end{array}$ & $\begin{array}{c}\text { Ortho } \\
\text { rhombic }\end{array}$ & $\begin{array}{l}\text { Tetra } \\
\text { gonal }\end{array}$ & Cubic \\
\hline (a) & Order & Disorder & Disorder & Disorder \\
\hline (b) & Order & Order & Disorder & Disorder \\
\hline (c) & Order & Order & Order & Disorder \\
\hline
\end{tabular}

FIG. 4. Scheme of the 12 order/disorder combinations, as a function of the crystalline directions and the long-range symmetry phases, allowed by the model of Comès et al. $[19,20]$. This very same diagram is found to hold also for the KLTN macroscopic response (see Fig. 3). 
compound ferroelectrics [46] suggest methods to evaluate the energy associated with the reorientation of the dipolar structures specifically associated with disorder. In this vein it may be possible to shed light on the mechanisms linking the inherent dipoles and the larger scale dipolar systems by analyzing the scaling of the reorientation energy as a function of structure size.

\section{Order/disorder transitions}

The features of $s(T)$ in correspondence of the phase transitions give information on their nature; specifically the typical order-disorder two-opposite-peaks line shape (as schematically depicted in Fig. 1) is exhibited for the three considered transitions: cubic/tetragonal ( $a, b, c$ directions), tetragonal/orthorhombic ( $a, b$ directions), and orthorhombic/rhombohedral ( $a$ direction). We may therefore conclude that all the long-range symmetry transformations have an order/disorder component. We observe that on the contrary, and in agreement with the Fröhlich treatment, in the case of order/order transformations the $s(T)$ curve exhibits only a positive peak (Fig. 3, $b$ and $c$ directions).

Significantly, when a single global phase is examined, the comparison of the disorder $(s<0)$ levels between the three directions shows significant differences. This behavior, in spite of the considerable qualitative agreement, quantitatively does not agree with the eight-sites model, which in fact is solely referred to an ideal $\mathrm{ABO}_{3}$ lattice. The Fröhlich entropy evolution reported in Fig. 3 shows that, for the KLTN, in the cubic phase, for a given temperature $T$, the negative amount of $s(T)$ along the $a$ direction is higher than that along the $b$ direction and even higher than along the $c$ direction. This contrasts with the nominally centrosymmetric nature of this phase, where the local eight-sites model, assigning to the three directions the same probability for the $B$-cation displacements, predicts the same amount of disorder along all crystalline axes. Analogous considerations hold for the tetragonal phase (disorder levels in $a$ and $b$ directions). Cubic phase anisotropy (Fig. 3) itself gives evidence of a strong symmetry breaking and supports the interpretation of this phenomenon as due to a strain-triggered flexoelectric effect [54]. On the other hand, in the tetragonal phase, the different level of disorder in the $a$ and $b$ directions, perpendicular to the preferential $c$ axis, signals a breaking of the nominal tetragonal symmetry. This symmetry breaking indicates that the crystal responds as a single domain, even though it has not been previously poled nor subjected to a coercive field during measurement.

\section{CONCLUSIONS}

Dielectric measurements on a solid-solution disordered ferroelectric single-crystal KLTN are performed in the temperature range $25-320 \mathrm{~K}$, along the three main crystalline directions for different frequencies. Permittivity measurements as a function of temperature $\epsilon(T)$ identify three phase transitions of a diffusive type at the critical temperatures $T_{C}=282,202$, and $156 \mathrm{~K}$, indicating the transformations of the (nominal) phases cubic-to-tetragonal, tetragonal-toorthorhombic, and orthorhombic-to-rhombohedral, respectively, upon cooling. Summarizing, we here provide:

(1) An experimental investigation of the macroscopic order-disorder features performed on a chemical substituted ferroelectric solid solution (KLTN single crystals), on the basis of the relationship between the permittivity behavior vs temperature and the entropy variation due to applied probing field (Fröhlich entropy).

(2) The analysis of the macroscopic order/disorder states with respect to the four phases and the three considered crystalline directions, which agree with the 12 combinations predicted by the Comès pattern, finds that behavior agrees with the microscopic eight-sites model, which holds for nominally pure perovskite crystals. Congruently Fröhlich entropy analysis signals that the lowest-temperature rhombohedral phase is the only ordered stable symmetry of our system. Furthermore the analysis of the Fröhlich entropy evolution in correspondence of the transitions fully confirms the orderdisorder character of all the phase transformations.

(3) The analysis of the permittivity response and Fröhlich entropy for the four long-range symmetry phases in relation to the crystalline directions, which points out marked anisotropic characteristics: in a bulk crystal the coexistence of macroscopic states having different ordering in different directions for a single crystalline phase is evidenced in an unpoled sample.

(4) Evidence of the breaking of the macroscopic centric symmetry in the nominally cubic phase in absence of strains due to external electric field or forces. The breaking of macroscopic symmetry is found also in the tetragonal phase with respect to its axis. This "self-poling" is attributed to a microscopic strain-driven flexoelectric effect that is known to play a relevant role in ferroelectrics.

From a methodological point of view, our study underlines how Fröhlich entropy forms a powerful method complementary to the usual experimental techniques based on local investigation at fixed temperatures, e.g., x ray, electron, neutron diffraction, and EXAFS, XANES. Specifically, the ability to analyze response in different macroscopic directions makes it suitable to investigate systems which are inhomogeneous in chemical composition and strain disposition (including anisotropic features), as occurs, e.g., in complex relaxors and ferroelectrics, where order-disorder states typically emerge as a function of temperature, crystal phases, and directions.

\section{ACKNOWLEDGMENTS}

This work was partially founded by Grants PHOCOSRBFR08E7VA and PRIN 2014BFNWZ2 of the Italian Ministry of Research. We thank L. Cattaneo for technical assistance.
[1] T. Siegrist, P. Jost, H. Volker, M. Woda, P. Merkelbach, C. Schlokermann, and M. Wuttig, Disorder-induced localization in crystalline phase-change materials, Nat. Mater. 10, 202 (2011).
[2] A. V. Ievlev, S. Jesse, A. N. Morozovska, E. Strelcov, E. A. Eliseev, Y. V. Pershin, A. Kumar, V. Ya. Shur, and S. V. Kalinin, Intermittency, quasiperiodicity and chaos in probe-induced ferroelectric domain switching, Nat. Phys. 10, 59 (2013). 
[3] M. J. Polking, M. G. Han, A. Yourdkhani, V. Petkov, C. F. Kisielowski, V. V. Volkov, Y. Zhu, G. Caruntu, A. P. Alivisatos, and R. Ramesh, Ferroelectric order in individual nanometrescale crystals, Nat. Mater. 11, 700 (2012).

[4] N. Balke, S. Choudhury, S. Jesse, M. Huijben, Y. H. Chu, A. P. Baddorf, L. Q. Chen, R. Ramesh, and S. V. Kalinin, Deterministic control of ferroelastic switching in multiferroic materials, Nat. Nanotechnol. 4, 868 (2009).

[5] A. Bokov, Recent progress in relaxor ferroelectrics with perovskite structure, J. Mater. Sci. 41, 31 (2006).

[6] G. Biroli, In search of the perfect glass, Nat. Phys. 10, 555 (2014).

[7] D. S. Wiersma, Disordered photonics, Nature Photon. 7, 188 (2013).

[8] M. Li, M. J. Pietrowski, R. A. De Souza, H. Zhang, I. M. Reaney, S. N. Cook, J. A. Kilner, and D. C. Sinclair, A family of oxide ion conductors based on the ferroelectric perovskite $\mathrm{Na}_{0.5} \mathrm{Bi}_{0.5} \mathrm{TiO}_{3}$, Nat. Mater. 13, 31 (2013).

[9] A. I. Khan, K. Chatterjee, B. Wang, S. Drapcho, L. You, C. Serrao, S. R. Bakaul, R. Ramesh, and S. Salahuddin, Negative capacitance in a ferroelectric capacitor, Nat. Mater. 14, 182 (2014).

[10] E. DelRe, F. Di Mei, J. Parravicini, G. B. Parravicini, A. J. Agranat, and C. Conti, Subwavelength anti-diffracting beams propagating for more than 1000 Rayleigh lengths, Nature Photon. 9, 228 (2015).

[11] A. A. Bokov and Z.-G. Ye, Dielectric relaxation in relaxor ferroelectrics, J. Adv. Dielect. 2, 1241010 (2012).

[12] G. A. Samara, The relaxational properties of compositionally disordered $\mathrm{ABO}_{3}$ perovskites, J. Phys.: Condens. Matter 15, R367 (2003).

[13] V. V. Shvartsman and D. C. Lupascu, Lead-free relaxor ferroelectrics, J. Am. Ceram. Soc. 95, 1 (2012).

[14] Y. Chang, C. Wang, S. Yin, R. C. Hoffman, and A. G. Mott, Kovacs effect enhanced broadband large field of view electrooptic modulators in nanodisordered KTN crystals, Opt. Express 21, 17760 (2013).

[15] Y. Chang, C. Wang, S. Yin, R. C. Hoffman, and A. G. Mott, Giant electro-optic effect in nanodisordered KTN crystals, Opt. Lett. 22, 4574 (2013).

[16] W. Kleemann and R. Lindner, Dynamic behavior of polar nanodomains in $\mathrm{PbMg}_{1 / 3} \mathrm{Nb}_{2 / 3} \mathrm{O}_{3}$, Ferroelectrics 199, 1 (1997).

[17] P. B. Ishai, C. E. M. de Oliveira, Y. Ryabov, Yu. Feldman, and A. J. Agranat, Glass forming liquid kinetics manifested in a KTN:Cu crystal, Phys. Rev. B 70, 132104 (2004).

[18] J. Parravicini, C. Conti, A. J. Agranat, and E. DelRe, Programming scale-free optics in disordered ferroelectrics, Opt. Lett. 37, 2355 (2012).

[19] R. Comès, M. Lambert, and A. Guinier, The chain structure of $\mathrm{BaTiO}_{3}$ and $\mathrm{KNbO}_{3}$, Solid State Commun. 6, 715 (1968).

[20] R. Comès, M. Lambert, and A. Guinier, Désordre linéaire dans les cristaux (cas du silicium, du quartz, et des pérovskites ferroélectriques), Acta Crystallogr. Sect. A 26, 244 (1970).

[21] W. Kleemann, Relaxor ferroelectrics: Cluster glass ground state via random fields and random bonds, Phys. Status Solidi B 251, 1993 (2014).

[22] D. Pierangeli, F. Di Mei, J. Parravicini, G. B. Parravicini, A. J. Agranat, and E. DelRe, Observation of an intrinsic nonlinearity in the electro-optic response of freezing relaxors ferroelectrics, Opt. Mater. Express 4, 1487 (2014).
[23] J. Toulouse, The three characteristic temperatures of relaxor dynamics and their meaning, Ferroelectrics 369, 203 (2008).

[24] G. Burns and F. H. Dacol, Crystalline ferroelectrics with glassy polarization behavior, Phys. Rev. B 28, 2527 (1983).

[25] A. Agranat, R. Hofmeister, and A. Yariv, Characterization of a new photorefractive material: $\mathrm{K}_{1-y} \mathrm{~L}_{y} \mathrm{~T}_{1-x} \mathrm{~N}_{x}$, Opt. Lett. 17, 713 (1992).

[26] F. Jona and G. Shirane, Ferroelectric Crystals (Dover, New York, 1993).

[27] K. Tsuda, R. Sano, and M. Tanaka, Nanoscale local structures of rhombohedral symmetry in the orthorhombic and tetragonal phases of $\mathrm{BaTiO}_{3}$ studied by convergent-beam electron diffraction, Phys. Rev. B 86, 214106 (2012).

[28] K. Tsuda, R. Sano, and M. Tanaka, Observation of rhombohedral nanostructures in the orthorhombic phase of $\mathrm{KNbO}_{3}$ using convergent-beam electron diffraction, Appl. Phys. Lett. 102, 051913 (2013).

[29] E. A. Stern, Character of Order-Disorder and Displacive Components in Barium Titanate, Phys. Rev. Lett. 93, 037601 (2004).

[30] B. Zalar, A. Lebar, J. Seliger, R. Blinc, V. V. Laguta, and M. Itoh, NMR study of disorder in $\mathrm{BaTiO}_{3}$ and $\mathrm{SrTiO}_{3}$, Phys. Rev. B 71, 064107 (2005).

[31] B. Zalar, V. V. Laguta, and R. Blinc, NMR Evidence for the Coexistence of Order-Disorder and Displacive Components in Barium Titanate, Phys. Rev. Lett. 90, 037601 (2003).

[32] B. Ravel, E. A. Stern, I. Vedrinski, and V. Kraizman, Local structure and the phase transitions of $\mathrm{BaTiO}_{3}$, Ferroelectrics 206, 407 (1998).

[33] D. La-Orauttapong, J. Toulouse, J. L. Robertson, and Z.-G. Ye, Diffuse neutron scattering study of a disordered complex perovskite $\mathrm{Pb}\left(\mathrm{Zn}_{1 / 3} \mathrm{Nb}_{2 / 3}\right) \mathrm{O}_{3}$ crystal, Phys. Rev. B 64, 212101 (2001).

[34] G. Xu, G. Shirane, J. R. D. Copley, and P. M. Gehring, Neutron elastic diffuse scattering study of $\mathrm{Pb}\left(\mathrm{Zn}_{1 / 3} \mathrm{Nb}_{2 / 3}\right) \mathrm{O}_{3}$, Phys. Rev. B 69, 064112 (2004).

[35] K. Hirota, S. Wakimoto, and D. E. Cox, Neutron and x-ray scattering studies of relaxors, J. Phys. Soc. Jpn. 75, 111006 (2006).

[36] R. Pirc and Z. Kutnjak, Electric-field dependent freezing in relaxor ferroelectrics, Phys. Rev. B 89, 184110 (2014).

[37] U. Aschauer, R. Pfenninger, S. M. Selbach, T. Grande, and N. A. Spaldin, Strain-controlled oxygen vacancy formation and ordering in $\mathrm{CaMnO}_{3}$, Phys. Rev. B 88, 054111 (2013).

[38] E. DelRe, E. Spinozzi, A. J. Agranat, and C. Conti, Scale-free optics and diffractionless waves in nanodisordered ferroelectrics, Nature Photon. 5, 39 (2011).

[39] J. Parravicini, F. Di Mei, C. Conti, A. J. Agranat, and E. DelRe, Diffraction cancellation over multiple wavelengths in photorefractive dipolar glasses, Opt. Express 19, 24109 (2011).

[40] J. Parravicini, A. J. Agranat, C. Conti, and E. DelRe, Rejuvenation in scale-free optics and enhanced diffraction cancellation life-time, Opt. Express 20, 27382 (2012).

[41] H. Fröhlich, Theory of Dielectrics (Clarendon, Oxford, 1958).

[42] B. K. P. Scaife, Principles of Dielectrics (Clarendon, Oxford, 1998).

[43] G. B. Parravicini, A. Stella, P. Tognini, P. G. Merli, A. Migliori, P. Cheyssac, and R. Kofman, Insight into the premelting and melting processes of metal nanoparticles through capacitance measurements, Appl. Phys. Lett. 82, 1461 (2003). 
[44] J. Parravicini, A. J. Agranat, C. Conti, and E. DelRe, Equalizing disordered ferroelectrics for diffraction cancellation, Appl. Phys. Lett. 101, 111104 (2012).

[45] I. Segal, E. Dorfman, O. Yoffe, I. Bezrukavnikov, and A. J. Agranat, Direct ICP-OES determination of $\mathrm{Cu}, \mathrm{Fe}, \mathrm{Li}$, Ti, and $\mathrm{V}$ in potassium lithium tantalate niobate crystals, Atom. Spectros. 21, 46 (2000).

[46] C. Stock, P. M. Gehring, G. Xu, D. Lamago, D. Reznik, M. Russina, J. Wen, and L. A. Boatner, Fluctuating defects in the incipient relaxor $\mathrm{K}_{1-x} \mathrm{Li}_{x} \mathrm{TaO}_{3}(x=0.02)$, Phys. Rev. B 90, 224302 (2014).

[47] A. Sassella, D. Braga, M. Campione, T. Ciabattoni, M. Moret, J. Parravicini, and G. B. Parravicini, Probing phase transitions and stability of organic semiconductor single crystals by dielectric investigation, J. Appl. Phys. 109, 013529 (2011).

[48] G. B. Parravicini, M. Campione, F. Marabelli, M. Moret, and A. Sassella, Experimental assessment of nonergodicity in tetracene single crystals, Phys. Rev. B 86, 024107 (2012).

[49] J. Fousek, L. E. Cross, and D. B. Litvin, Possible piezoelectric composites based on the flexoelectric effect, Mater. Lett. 39, 287 (1999).
[50] J. Y. Fu and L. E. Cross, On the flexoelectric effects in solid dielectrics: Theories and applications, Ferroelectrics 354, 238 (2007).

[51] J. Y. Fu, W. Zhu, N. Li, N. B. Smith, and L. E. Cross, Gradient scaling phenomenon in microsize flexoelectric piezoelectric composite, Appl. Phys. Lett. 91, 182910 (2007).

[52] L. E. Cross, Relaxor ferroelectrics, Ferroelectrics 76, 241 (1987).

[53] G. A. Samara and E. L. Venturini, Ferroelectric/relaxor crossover in compositionally disordered perovskites, Phase Transit. 79, 21 (2006).

[54] A. Biancoli, C. M. Fancher, J. L. Jones, and D. Damjanovic, Breaking of macroscopic centric symmetric in paraelectric phases of ferroelectric materials and implications for flexoelectricity, Nat. Mater. 14, 224 (2014).

[55] R. Pattnaik and J. Toulouse, New Dielectric Resonances in Mesoscopic Ferroelectrics, Phys. Rev. Lett. 79, 4677 (1997).

[56] S. Prosandeev, D. Wang, A. R. Akbarzadeh, B. Dkhil, and L. Bellaiche, Field-Induced Percolation of Polar Nanoregions in Relaxor Ferroelectrics, Phys. Rev. Lett. 110, 207601 (2013). 